\title{
Smartphones for Smart Wheelchairs
}

\author{
Aleksandar Milenković, Mladen Milosevic, Emil Jovanov \\ Electrical and Computer Engineering Department \\ The University of Alabama in Huntsville \\ Huntsville, AL, U.S.A. \\ \{milenka,mladen.milosevic,ejovanoe\}@uah.edu
}

\begin{abstract}
Individuals with limited ambulatory skills are at high risk for all physical inactivity-related diseases, such as coronary disease and diabetes. Increased physical activity can significantly lower risks of these diseases. However, quantifying recommendations for increased physical activity remain challenging for individuals who use wheelchairs for mobility. In this paper we introduce a smart wheelchair that utilizes a smartphone with its built-in sensors to capture and record physical activity of manual wheelchair users in both unstructured and structured environments. We develop algorithms for data acquisition and processing on the smartphone and implement them in an Android application called mWheelness. The application is successfully tested in laboratory and free-living experiments using several modern smartphones.
\end{abstract}

Keywords-mobile computing, body sensor networks, health monitoring, wheelchairs.

\section{INTRODUCTION}

Public health groups have been advocating for an increase in physical activity levels as a way to lower the risk of inactivity-related diseases, such as cardiovascular disease, insulin resistance, hyperglycemia, and type 2 diabetes. Physically inactive individuals are almost twice as likely to develop coronary heart disease when compared to those who exercise regularly. Recent estimates suggest that the impact of physical inactivity on mortality risk is approaching tobacco as one of the leading causes of death in the ablebodied population [1].

People with limited ambulatory skills who use wheelchairs for mobility are especially at high-risk for all inactivity-related diseases. For example, it has been reported that a person with a spinal cord injury (SCI) has a significantly greater risk of mortality from coronary heart disease $(225 \%)$ than an able-bodied person [1]. According to a 2005 U.S. Census Bureau's Survey, over 3.3 million Americans use some type of wheelchairs for mobility and with aging population this number is likely to continue to grow.

Many organizations developed specific physical activity recommendations for disabled adults. The U.S. Department of Health and Human Services recommends that disabled adults should accumulate 150 minutes per week of moderateintensity and 75 minutes per week of vigorous-intensity aerobic activity [2]. To quantify compliance with these recommendations, a method that can accurately estimate physical activity for this group of individuals is needed.

There have been a number of studies focusing on activity monitoring in the able-bodied population that rely on step counters and other accelerometer-based systems or on surveys [3], [4]. However, despite having a variety of different methods to utilize for the measurement of physical activity, each has its own limitations for manual wheelchair users. For example, pedometers and other step activity monitors will not apply to individuals who are unable to walk. Several studies focus on suitability of accelerometers for assessment of physical activity of individuals in wheelchairs [5]-[7], however they often require expensive and sophisticated wheelchair monitors and/or user instrumentation with wearable electronics that may impede normal daily activities. Alternatively, surveys are commonly used for the elderly and even individuals with disabilities. However, there are inherent problems with subjectivity and inaccuracy from recall and interpretation. This is of special concern for individuals who use wheelchairs because they often spend the majority of time in low-intensity activities. We know that individuals using wheelchairs for mobility are some of the most sedentary people in society, but assessing physical activity and quantifying compliance with prescribed exercise regimens are highly constrained due to lack of affordable and reliable systems for physical activity monitoring. Therefore, the development of affordable, unobtrusive, and easy-to-use systems to support assessment of physical activity of manual wheelchair users is of utmost importance.

Mobile computing devices such as smartphones, tablet computers, and e-readers have steadily been gaining market share, dethroning laptop and desktop computers as dominant personal computing platforms. According to an estimate for 2011, vendors shipped 487.7 million smartphones (up 63\% from the year before) [8]. It is forecast that the number of smartphones shipped in 2015 will reach 1.4 billion. Modern smartphones integrate a growing number of sensors, such as an accelerometer, magnetometer (digital compass), gyroscope, proximity, global positioning system (GPS), camera, microphone, ambient light, humidity, and temperature. Major mobile operating systems, such as Android, iOS, and Windows 8 support frameworks for managing the sensors, including continual sampling, thus enabling a wide variety of new applications in different 
domains, such as social networks, transportation, commerce, education, and entertainment. New companies emerged to offer wearable wireless biosensors targeting wellness applications [9], [10]. Convergence of smart biosensors, smartphones, and cloud services allows us to look beyond the existing fitness services and develop platforms for mobile health monitoring in unsupervised and unstructured environments such as continual monitoring during activities of daily living [11], [12].

To meet the challenge and provide an affordable, reliable, and easy to use solution for monitoring the physical activity of users who rely on wheelchairs for mobility we developed a smart wheelchair - a common wheelchair instrumented only with a smartphone that is used to track a user's physical activity. We utilized the smartphone's builtin sensors such as a magnetic sensor for monitoring wheelchair speed and distance traveled, an accelerometer for monitoring smartphone's orientation and wheelchair inclination, and a proximity sensor to determine whether the wheelchair is hand-propelled or pushed. In addition, we employ a wearable chest belt to monitor and record the user's heart activity and energy expenditure. A smartphone application called $\mathrm{mWheelness}$ collects the data from the sensors and performs periodic uploads to an mHealth server.

Our system enables collection of data about heart and physical activity during specialized rehabilitation sessions and during activities of daily living. Automatic uploads of monitored sessions into server databases enable healthcare professionals to quantitatively assess level and type of user's activity when they return home; a critical component missing in current practices. With quantitative assessment of exercise, healthcare professionals can verify user's compliance with prescribed exercise regimen, and can better understand correlation between exercise intensity and recovery. They can also assess the impact of new approaches for the treatment of people with inactivity-induced conditions. To the best of our knowledge this is the first system that relies exclusively on the built-in smartphone's sensors for quantifying physical activity of wheelchair users. By utilizing smartphones we provide an inexpensive assistive platform that is easy to use. The proposed system can significantly improve the function, wellness, and overall quality of life for people with limited ambulatory skills as well as their caregivers and families.

The rest of the paper is organized as follows. Section II describes the system view of the proposed system. Section III details wheelchair's instrumentation, methods, and algorithms used to quantify activity of wheelchair users. Section IV describes mWheelness application and Section V gives test results and concluding remarks.

\section{SYSTEM ARCHITECTURE}

The mWheelness infrastructure for monitoring activity of wheelchair users features a 3-tiered architecture with inertial and heart sensors at Tier 1, a smartphone at Tier 2, and an mHealth server at Tier 3 (Figure 1). The system can record, log, display, and communicate information about the user's physical and heart activity during normal daily activities or exercise sessions.
Tier 1 includes a wearable chest belt for monitoring heart activity that wirelessly connects to the smartphone. For monitoring the user's physical activity (e.g., speed and distance travelled) we rely solely on smartphone's built-in sensors, including a magnetic sensor, an accelerometer, and a proximity sensor. Tier- 2 consists of the mWheelness application that runs on the smartphone. The application supports (a) configuring, calibrating, and managing multiple sensors; (b) configuring exercise sessions; (c) data retrieval and analysis from individual sensors to extract and log the user status information, and (d) user interface. The collected physical activity and health status information is recorded locally on the smartphone and can be periodically uploaded to the mHealth server over the Internet.

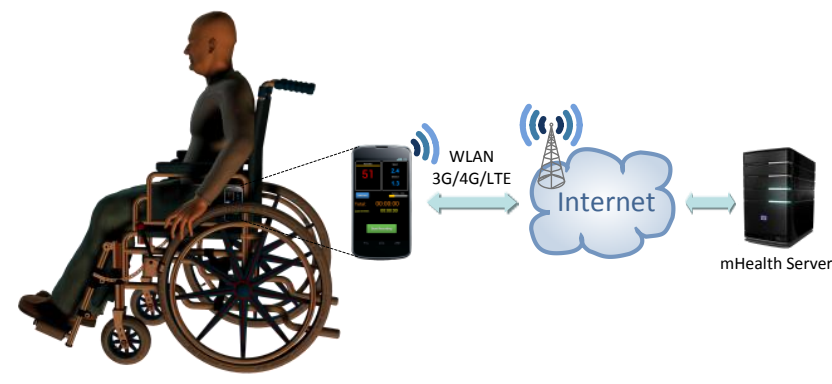

Figure 1. mWheelness: the system view.

\section{SMARTPHONE SENSORS FOR ACTIVITY MONITORING}

\section{A. Wheelchair insrumentation}

Figure 2 illustrates the proposed wheelchair instrumentation with a smartphone. The smartphone is placed in a holder on a side of the wheelchair. A small magnet is attached on a wheel of the wheelchair. The smartphone's magnetic sensor is sensing the Earth magnetic field and is normally used to aid navigation by determining the Earth's magnetic poles and the smartphone's orientation. The magnetic sensor senses the $x, y$, and $z$ components of the magnetic field as illustrated in Figure 2 (the actual orientation may vary across different smartphones). By placing a small magnet on the wheel, we induce a change in the magnetic field sensed by the magnetic sensor of the smartphone when the magnet moves over the smartphone. This change produces a characteristic signature in the magnetic field signals that can be sensed, recorded, and processed on the smartphone. By processing the magnetic field signals we can detect and timestamp an event - when the magnet moves right over the smartphone which corresponds to one revolution of the wheelchair's wheel.

A smartphone's accelerometer measures proper acceleration and is typically used to keep the screen upright regardless of the smartphone orientation. In our setup we process the $x, y$, and $z$ acceleration components to determine smartphone's orientation, i.e., whether it is placed in the wheelchair holder or not. Activity recording is enabled only when the smartphone is properly mounted on the wheelchair. In addition, the accelerometer data is used to determine slope 
of the wheelchair which can further be used to determine vertical gain and loss during exercise.

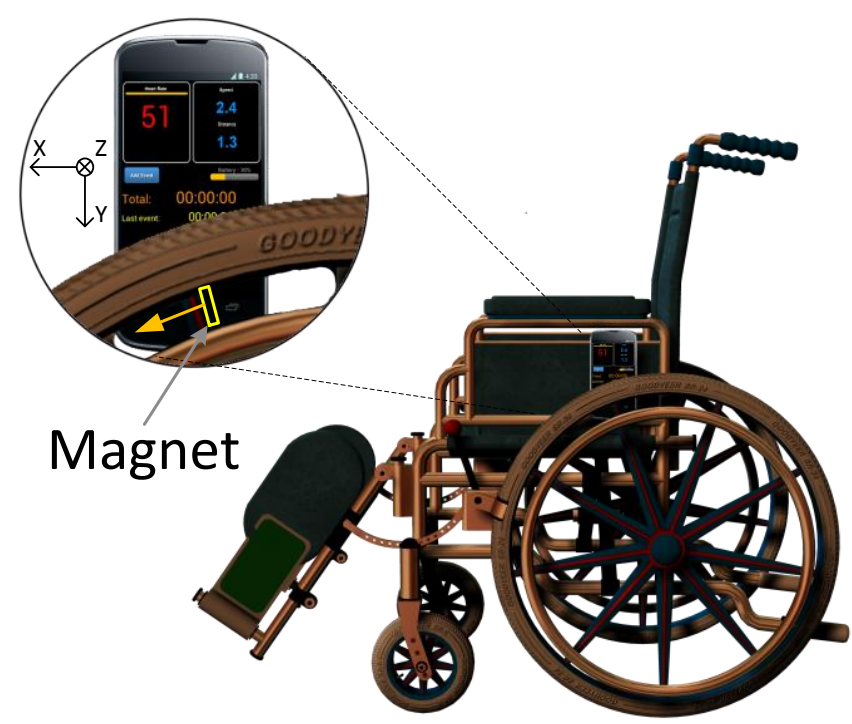

Figure 2. Smartphone instrumentation of a wheelchair.

A smartphone's proximity sensor is typically used to determine when the smartphone is brought up to the user's ear and usually acts as a binary sensor. In our deployment, the smartphone's proximity sensor is used to determine whether the user hand-propels the wheelchair or it is pushed. This information can be used to further qualify the user's activity.

The following subsections describe the sensing and algorithms for data processing used to determine the parameters of interest for activity monitoring.

\section{B. Measuring speed and distance}

Figure 3 shows the raw magnetic field components recorded by our custom application running on a smartphone mounted on a wheelchair as shown in Figure 2. The wheelchair is placed on a treadmill that moves at the constant speed of 1 mile per hour (mph) (Figure 3a), $2 \mathrm{mph}$ (Figure $3 \mathrm{~b}$ ), and $4 \mathrm{mph}$ (Figure 3c). Whereas all three axes of the magnetic field $(x, y$, and $z$ ) show a distinct signature when the magnet moves over the smartphone, the changes in the $z$ component are the most distinct. Consequently, our algorithm for detection of a wheel revolution focuses on this component of the magnetic field. The signal is such that even a simple visual inspection may be used to confirm the feasibility of the proposed approach - changes in the magnetic field induced by the magnet can indeed be used to determine the number of wheel revolutions. For example, we can identify 5 peaks in the $z$ component of the magnetic field during a window of 20 seconds (Figure 3a). This corresponds to 5 revolutions of a 24" diameter wheel, which in turn corresponds to $\sim 1 \mathrm{mph}$ speed. Similarly, we can identify 19 peaks when the treadmill belt moves at $4 \mathrm{mph}$.

Whereas the treadmill experiment confirms feasibility of the proposed approach, it presents somewhat idealized conditions. The wheelchair is fixed on the treadmill and its position relative to the Earth's magnetic field remains unchanged during the experiment. In real-world conditions the wheelchair will constantly change its position relatively to the Earth's magnetic field. This will result in changing the base values of the magnetic field observed by the smartphone's magnetic sensor.

Figure $4 \mathrm{a}$ shows the $z$ component of the magnetic field recorded during a freewheeling experiment when a user propels himself in a wheelchair moving through a squareshaped hallway, making a full round trip of approximately 100 meters in length. We can observe a slow-changing DC component of the magnetic field due to changes in the smartphone position relative to the Earth's magnetic field. Fortunately, the spikes induced by the proximity of the magnet on the wheel when the magnet moves over the smartphone can still be easily detected.

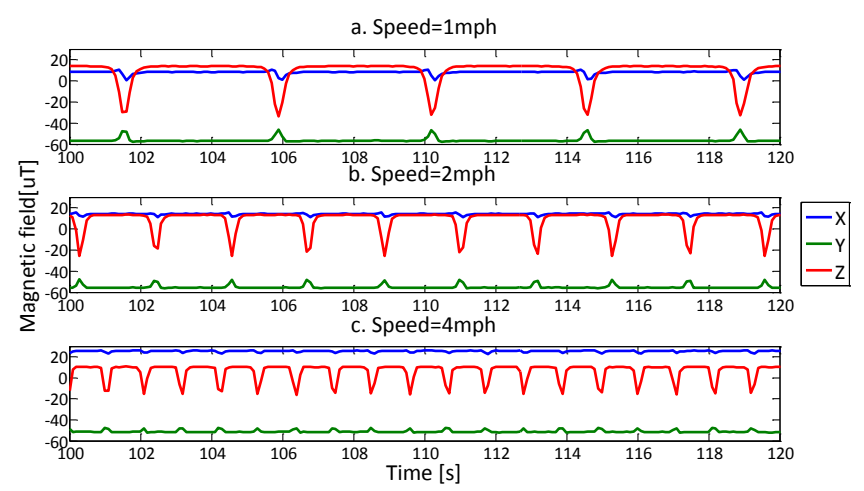

Figure 3. Smartphone recordings of the $\mathrm{x}, \mathrm{y}$, and $\mathrm{z}$ components of the magnetic field for different wheelchair speeds: (a) $1 \mathrm{mph}$; (b) $2 \mathrm{mph}$; and (c) $4 \mathrm{mph}$.

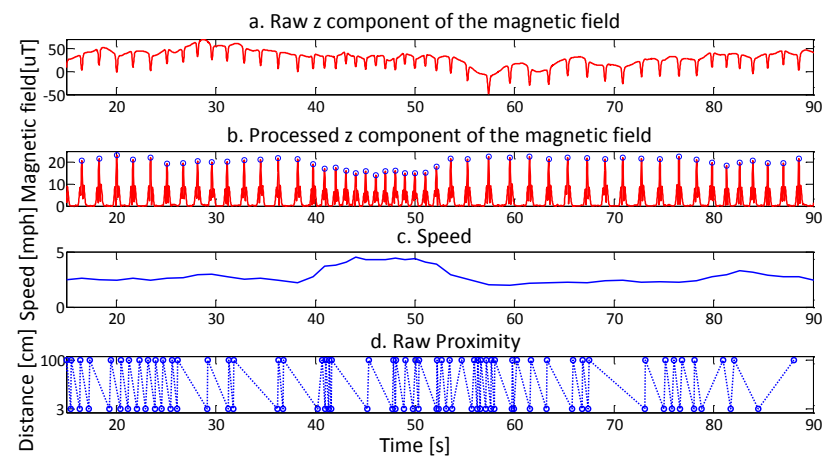

Figure 4. From signals to information: (a) $z$ component of the magnetic field; (b) processed $z$ component; (c) calculated speed; and (d) proximity sensor data.

The next step is to transform the original signal representing the $z$ component of the magnetic field into a signal that allows for efficient detection of peaks. By detecting and time-stamping peaks in the transformed signal, we can precisely derive the wheelchair speed and distance traveled. In developing the signal processing algorithm our goal is to achieve accuracy and reliability at minimum cost in computation time and energy consumed. 
The transformation should remove a slow changing component caused by changes in the smartphone orientation relative to the Earth's magnetic field and amplify the component of the signal caused by the proximity of the magnet on the wheel. An incoming sample, $z(i)$, is stored in a sliding buffer that keeps a window of 40 most recent samples - this corresponds to 1 second in time with the sampling frequency of $40 \mathrm{~Hz}$. Note: in general the window size is a function of the sampling frequency. The processed signal, $p z$, is calculated as shown in (1). Figure $4 \mathrm{~b}$ shows the transformed signal.

$$
p z(i)=\left|z(i)-\frac{1}{40} \sum_{j=-19}^{j=20} z(i+j)\right|
$$

The second step is to detect peaks in the processed signal $p z$. Our algorithm for peak detection involves a state machine with three states, Pre-Peak, Expect-Peak, and Post-Peak. Pre-Peak is maintained as long as the processed signal samples, $p z(i)$, are below a certain threshold. When a sample is above the threshold we enter the Expect-Peak state. The algorithm exits the Expect-Peak state once the processed samples are below the threshold. The threshold is set at 13 $\mu \mathrm{T}$. In this state the samples are buffered and searched for the maximum. The sample number of the maximum is used to precisely timestamp the moment when the peak occurred. To avoid false positives, Post-Peak is entered. The state machine remains in this state for a pre-defined period of time. The duration of the Post-Peak state is determined by the maximum speed we would like to detect. For example, at the speed of $10 \mathrm{mph}$, which is twice as the realistic maximum speed of $5 \mathrm{mph}$, the time distance between two peaks is 0.428 seconds for a wheelchair with 24 " wheel. This means that in normal operation no two valid peaks can possibly be detected in a period shorter than 0.428 seconds.

Figure $4 \mathrm{~b}$ shows the transformed signal with blue circles identifying peaks detected by the proposed algorithm. The visual inspection indicates that algorithm achieves $100 \%$ accuracy. The algorithm has been extensively tested in both treadmill experiments and in hand-propelled wheelchair experiments.

Measuring speed and distance travelled can easily be derived from the sequence of time-stamped peaks. Let us assume a sequence of peak timestamps $t_{i-1}, t_{i}, t_{i+l}$. The speed at time $t_{i}, v\left(t_{i}\right)$, is calculated as shown in (2), where $d$ is the diameter of the wheel. If no peak is detected during a predefined time window (e.g., 8 seconds), the speed is reset to zero. Figure $4 \mathrm{c}$ shows the calculated speed over time for the free-wheeling experiment. The distance travelled, $D$, is maintained by simply counting the number of detected revolutions, $N$, and can be calculated using (3).

$$
\begin{aligned}
& v\left(t_{i}\right)=\frac{\pi \mathrm{d}}{t_{i}-t_{i-1}} \\
& D=\pi \mathrm{dN}
\end{aligned}
$$

In designing the peak detection algorithm and speed estimation we need to consider the sampling frequency of the smartphone's magnetic sensor. Higher sampling frequency provides better resolution of timestamps and consequently more accurate speed estimation. Lower sampling frequency may reduce the compute complexity and storage requirements for the peak detection as well as the power consumption of the smartphone. In determining the sampling frequency we start from an application requirement - what is required accuracy for speed estimation. For example, if we want to achieve accuracy of $\Delta v=0.1 \mathrm{mph}$, the minimum required sampling frequency, $\mathrm{F}_{\mathrm{S}}$, for a given speed $v$ can be determined using (4). Thus, to distinguish between $0.9 \mathrm{mph}$ and $1 \mathrm{mph}$, the minimum sampling frequency is $2.1 \mathrm{~Hz}$ (assuming a 24" wheel). If we want to distinguish between 3.9 and $4.0 \mathrm{mph}$, the minimum required sampling frequency is $36.4 \mathrm{~Hz}$. Finally, if we want to distinguish between 5.9 $\mathrm{mph}$ and $6.0 \mathrm{mph}$, the minimum sampling frequency is 82.6 $\mathrm{Hz}$. For all practical applications where wheelchair speed is rarely to exceed $4 \mathrm{mph}$, the sampling frequency of $40 \mathrm{~Hz}$ is adequate.

$$
F_{S}=\frac{1}{\frac{\pi \mathrm{d}}{v-\Delta v}-\frac{\pi \mathrm{d}}{v}}
$$

\section{Detection of hand propulsion}

The smartphone's proximity sensor can be utilized to determine whether the wheelchair is propelled by the user or possibly pushed by another person. A modern smartphone's proximity sensor typically reports only two states - an object is closer or farther than triggering distance of the sensor. Depending on the type of the sensor built in the smartphone, the triggering distance is typically $3-10 \mathrm{~cm}$. The proposed placement of the smartphone on the wheelchair makes it possible to detect hand propulsion - when the user places his/her hand on the hand rim of the wheel he/she will likely trigger the proximity sensor. When the wheelchair is pushed by another person, the user's hand is not likely to interfere with the smartphone's proximity sensor.

Our approach is to simply maintain a status of the proximity sensor: if it reported a change in a certain time window, we consider the wheelchair to be hand-propelled by the user. Figure $4 d$ shows the raw values reported by the proximity sensor of a Motorola RAZR M smartphone during the free-wheeling experiment. The proximity sensor reports two distances, $3 \mathrm{~cm}$ and $100 \mathrm{~cm}$. Frequent changes of the reported distance indicate hand propulsion during the entire experiment.

Whereas the proposed algorithm works well in our initial studies, it has its limitations - the user's hand may not always be close enough to trigger the proximity sensor and detection strongly depends on triggering distance of the proximity sensor. An alternative approach, albeit costlier in terms of processing and storage requirements, is to process still images or video captured by the smartphone camera in order to detect whether the user hand propels the wheelchair.

\section{Tilt and Incline Calculation}

Tracking of physical activity is conditioned by the smartphone being in the upright position in the wheelchair holder. This can be detected by calculating the smartphone 
tilt as shown in Figure 5a. The angle $\phi$ can be estimated as in (5). The tilt of the wheelchair in normal operation is unlikely to exceed a certain range (e.g., -10 to +10 degrees) and we use this property to determine whether the smartphone is in the holder or not. If this angle exceeds a certain predefined range, we consider the smartphone to be taken by the user, and the processing of the magnetometer signals for detection of wheel revolutions is suspended.

In a similar fashion we calculate the smartphone slope as shown in Figure 5b. Whereas the wheelchair propulsion will have an impact on the $x$ and $z$ components of the acceleration, $A_{x}$ and $A_{z}$ respectively, the $x$ and $y$ components of the acceleration, $A_{z}$ and $A_{y}$, will still be dominated by the Earth's gravity. By filtering the calculated angle $\theta(5)$, we can extract the slope of the wheelchair and thus use this information to determine the vertical gain and drop during an exercise session. An alternative approach to eliminate the impact of forces due to propelling is to consider only acceleration samples when the square root of the sum of squared acceleration components is very close to the Earth's gravity $\left(9.81 \mathrm{~m} / \mathrm{s}^{2}\right)$.

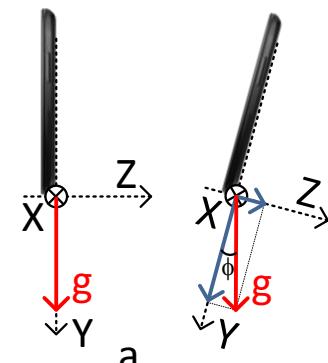

a.

Figure 5. Tracking tilt (a) and incline (b).

$$
\phi=\arctan \left(\frac{A_{z}}{\sqrt{A_{y}^{2}+A_{x}^{2}}}\right), \theta=\arctan \left(\frac{A_{x}}{\sqrt{A_{y}^{2}+A_{z}^{2}}}\right)
$$

The described algorithms for determining the smartphone's tilt and incline assume a perfect alignment of the wheelchair holder - angles $\phi$ and $\theta$ are both equal to 0 degrees when the wheelchair is on flat surface as shown in Figure 5. To accommodate for imperfect holder placement, we go through a calibration process where we calculate offsets $\phi_{\mathrm{o}}$ and $\theta_{\mathrm{o}}$, and the measured angles are compensated for these offsets.

An alternative approach to determining that the smartphone is in the wheelchair holder is to place an inexpensive NFC tag in the holder. The NFC capable smartphones can utilize this tag to detect when the smartphone is placed in the holder. In addition, this event can be used to automatically start the smartphone application.

\section{SMARTPHONE APPLICATION}

The mWheelness smartphone application implements the algorithms described in the previous section to support activity monitoring and logging. In addition, it supports monitoring and logging of heart activity. The recorded logs of registered users can optionally be uploaded to the mHealth databases.

Figure 6 shows characteristic screens of mWheelness. A configuration screen shown on the left allows a user to enter his/her gender, weight, age, wheel diameter, as well as the sampling rate, and the magnetic sensor detection threshold. In addition, the screen allows for configuring what type of information will be logged into a file on the smartphone and/or uploaded to the databases. It also guides the user through a calibration process providing acoustic clues to start calibration, place the smartphone in the holder, and remove it after the calibration is performed.

The user starts recording physical activity and heart activity by pressing the Start Recording button - please note that processing of the signals from the magnetic sensor will not start before the smartphone is in the upright position. During an exercise session mWheelness displays current inclination, speed, and distance travelled. In addition, it displays information about heart activity.
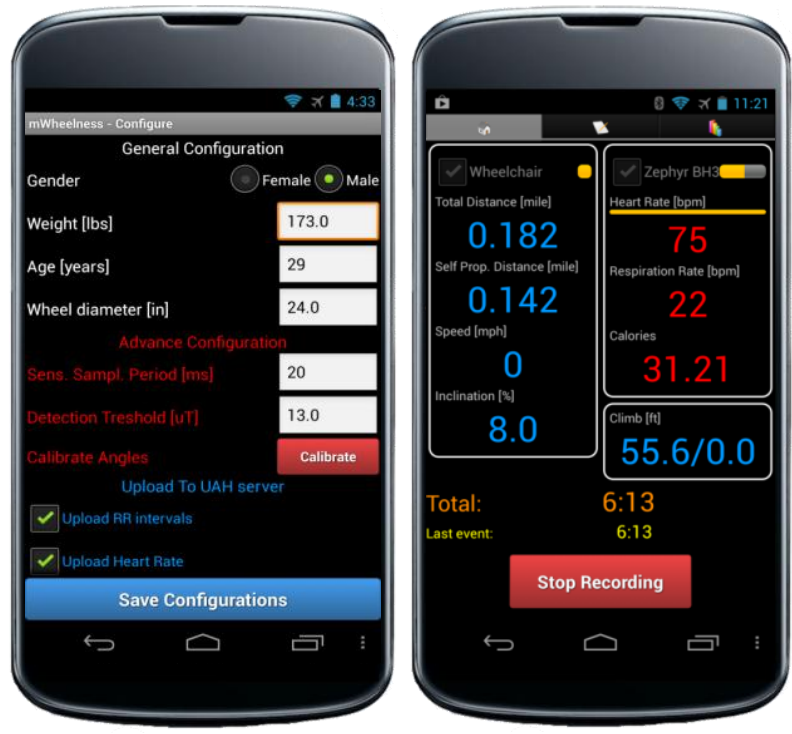

Figure 6. mWheelness Android application screens.

To monitor the user's heart activity we employ a commercially available Zephyr HXM heart monitor [9] that can be paired with the smartphone over Bluetooth. The monitor is mounted on a chest belt with textile electrodes. It sends a message every second containing the average heart rate and time stamps of the last 15 heart beats. mWheelness estimates energy expenditure in calories, $\Delta \mathrm{C}$, using (6) for men and (7) for women as proposed by Keytel et. al. [13]. It is a function of the heart rate (HR), the user's weight in kilograms $(\mathrm{W})$, and the age in years (A). We update the total energy every second $(\Delta \mathrm{T}=1 \mathrm{sec})$.

$$
\begin{array}{r}
\Delta \mathrm{C}=\frac{(-55.0969+0.6309 \cdot \mathrm{HR}+0.1988 \cdot \mathrm{W}+0.2017 \cdot \mathrm{A}) \cdot \Delta \mathrm{T}}{4.184} \\
\Delta \mathrm{C}=\frac{(-20.4022+0.4472 \cdot \mathrm{HR}-0.1263 \cdot \mathrm{W}+0.074 \cdot \mathrm{A}) \cdot \Delta \mathrm{T}}{4.184}
\end{array}
$$




\section{DISCUSSION AND CONCLUSIONS}

The mWheelness application is tested on several Android smartphones in controlled and free-living conditions. The controlled experiments are conducted on a treadmill while varying speed and inclination. The distance traveled and inclination reported by the application are compared against the corresponding parameters reported by the treadmill. mWheelness can run in a special mode that has additional support for logging all raw samples from the smartphone sensors. This allows us to verify inner workings of our algorithms offline - the raw data are processed in Matlab to generate events that are compared against the events logged by mWheelness.

Free-wheeling experiments involved five able-bodied individuals hand propelling the wheelchair on a rectangularshaped course of 201 meters in length. As test platforms we used Motorola RAZR M and HTC One X. Each individual completed the course two times, once in each direction. The average distance and standard deviation reported by mWheelness is $198.8 \pm 3.1$ meters for Motorola RAZR M and $200 \pm 3$ meters for HTC One X. This is an excellent result if we know that the maximum precision we can achieve is within two circumferences of the wheel from the course length (3.8 meters in our case). The RAZR M's proximity sensor with triggering distance of $3 \mathrm{~cm}$ occasionally misses hand movements during hand propelling for some users, whereas the HTC One X's proximity sensor with triggering distance of $9 \mathrm{~cm}$ performed flawlessly.
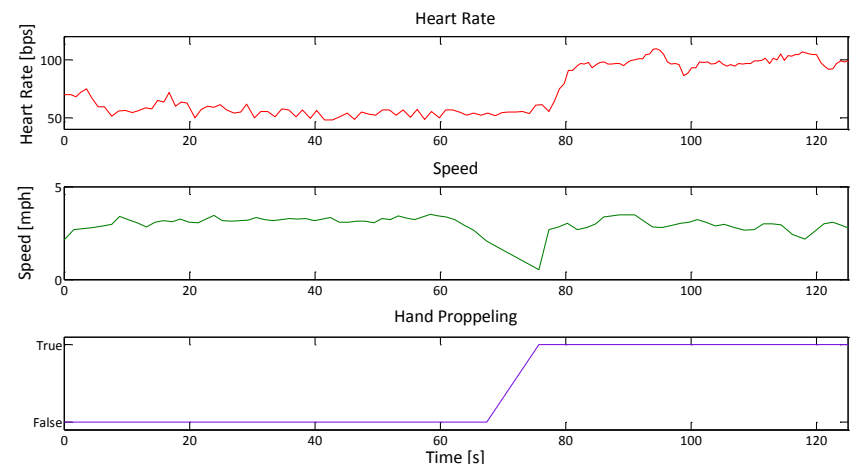

Figure 7. Heart Rate, Speed, and Hand Propelling during a freewheeling experiment

Figure 7 shows user's heart activity, wheelchair speed, and hand propelling flag recorded by mWheelness during an experiment. The wheelchair is pushed for the first 75 seconds, and hand propelled by the user afterwards. We can observe a steep increase in heart rate once the user started hand propelling.

To estimate maximum operating time of the mWheelness application with a single battery charge, we conducted several experiments on Motorola RAZR M. The operating time when only physical activity is logged (with WLAN,
Bluetooth, and mobile data turned off) is slightly over 20 hours. The operating time when both physical and heart activity are logged (with WLAN and mobile data turned off, Bluetooth is on) is slightly over 18 hours. The entire system is currently undergoing testing in a local rehabilitation hospital in both supervised and free-living conditions.

\section{ACKNOWLEDGMENT}

This work was supported in part by U.S. National Science Foundation under grant CNS-1205439. We are grateful to Ms. Allison Lindsey and Ms. Susan Creekmore from the Healthsouth Rehab Hospital, Huntsville, AL for their generous help in defining requirements for the wheelchair monitoring application.

\section{REFERENCES}

[1] P. L. Jacobs and M. S. Nash, "Modes, benefits, and risks of voluntary an delectrically induced exercise in persons with spinal cord injury.," J Spinal Cord Med, vol. 24, no. 1, pp. 10-8, 2001.

[2] U.S. Department of Health and Human Services, "2008 Physical Activity Guidelines for Americans.” [Online]. Available: http://www.health.gov/paguidelines/guidelines/default.aspx. [Accessed: 27-Jan-2013].

[3] D. Hendelman, K. Miller, C. Baggett, E. Debold, and P. Freedson, "Validity of accelerometry for the assessment of moderate intensity physical activity in the field," Medicine \& Science in Sports \& Exercise, vol. 32, no. Supplement, pp. S442-S449, Sep. 2000.

[4] G. J. Welk, J. A. Differding, R. W. Thompson, S. N. Blair, J. Dziura, and P. Hart, "The utility of the Digi-walker step counter to assess daily physical activity patterns," Med Sci Sports Exerc, vol. 32, no. 9 Suppl, pp. S481-488, Sep. 2000.

[5] R. Washburn and A. G. Copay, "Assessing Physical Activity During Wheelchair Pushing: Validity of a Portable Accelerometer,' Adapted Physical Activity Quaterly, vol. 16, no. 3, pp. 290 - 299, Jul. 1999.

[6] C. A. Warms and B. L. Belza, "Actigraphy as a measure of physical activity for wheelchair users with spinal cord injury," Nurs Res, vol. 53, no. 2, pp. 136-143, Apr. 2004.

[7] S. V. Hiremath and D. Ding, "Evaluation of activity monitors to estimate energy expenditure in manual wheelchair users," Conf Proc IEEE Eng Med Biol Soc, vol. 2009, pp. 835-838, 2009.

[8] "Smart phones overtake client PCs in 2011 | Canalys." [Online]. Available: http://www.canalys.com/newsroom/smart-phonesovertake-client-pcs-2011. [Accessed: 01-Jun-2012].

[9] "Zephyr." [Online]. Available: http://www.zephyrtechnology.com/. [Accessed: 21-Oct-2011].

[10] "Wahoo Fitness," 00:35:13. [Online]. Available: http://www.wahoofitness.com/. [Accessed: 21-Oct-2011].

[11] E. Jovanov, A. Milenkovic, C. Otto, and P. C. de Groen, "A wireless body area network of intelligent motion sensors for computer assisted physical rehabilitation," Journal of NeuroEngineering and Rehabilitation, vol. 2, no. 1, p. 6, Mar. 2005.

[12] A. Milenkovic, C. Otto, and E. Jovanov, "Wireless sensor networks for personal health monitoring: Issues and an implementation," Computer Communications (Special issue: Wireless Sensor Networks: Performance, Reliability, Security, and Beyond), vol. 29, no. 13-14, pp. 2521-2533, 2006.

[13] L. Keytel, J. Goedecke, T. Noakes, H. Hiiloskorpi, R. Laukkanen, L. van der Merwe, and E. Lambert, "Prediction of energy expenditure from heart rate monitoring during submaximal exercise," Journal of Sports Sciences, vol. 23, no. 3, pp. 289-297, Mar. 2005. 